\title{
Generate Asian Games 2018 Mascot For Batik Motif with Neural Style Transfer
}

\author{
Rakhmi Khalida ${ }^{1}$ \\ Information Technology Department, University of Gunadarma, Depok, Indonesia ${ }^{1}$
}

\begin{abstract}
In the past, batik artists to create a batik motif required special skills and a long time because motif is the main element in batik, and the uniqueness of batik is from the motif itself. Recently computers were able to produce many ordinary images turned into works of art even producing a new motif variant product. The method used is neural transfer style based on CNN architecture. It has performed feature extraction on batik motifs and has produced variations of batik motifs from collaborations of existing batik motifs such as the kawung batik motif and Asian Games 2018 mascot image. Neural transfer style empowers people around the world to experiment with their own creativity seeing the importance of style transfer in the commercial arts world and how art exists in the real world Index Terms.
\end{abstract}

Keywords: CNN, Batik, Mascot, Silhouette.

\section{INTRODUCTION}

In the past, redrawing an image in a certain style required a trained artist and a lot of time. Since the mid-1990s, the art theories behind beautiful works of art have attracted the attention of not only artists but many computer science researchers. There are many literature studies and techniques that explore how to turn images into synthetic works of art one of which is non photorealistic rendering (NPR) [1] [2] [3] which is inspiring, and at that time was an area of research by the computer graphics community. However, this NPR structuring algorithm is designed for certain artistic styles [4] [5] and cannot be easily extended to other styles.

As the host of the 2018 Asian Games, of course, Indonesia is doing all the preparations well, one of which is the mascot. The mascots prepared include the diversity of cultures, languages and historical relics that are put together. With the value of diversity and unity, Indonesia introduced 3 mascots with different energies, representing the energy contained in the Asian Games.

The three mascots are Bhin Bhin (bird of paradise), Atung (Bawean deer) and Kaka (one-horned rhinoceros) which are equipped with traditional Indonesian batik. Bhin Bhin wore a vest with an Asmat motif from Papua. Atung wore a sarong with a tumpal motif from Jakarta. Kaka wears traditional clothes with typical Palembang flower motifs [6]. Fig 1 is the three mascots Asian Games 2018.

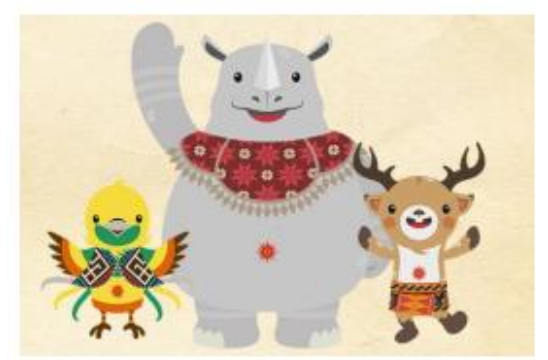

Fig. 1 The three mascots asian games 2018

Batik is a craft that has a high artistic value and has become part of Indonesian culture. Batik comes from the Javanese amba which means writing and dots. Batik means an image written on a cloth using batik wax or wax as a medium as well as a batik cloth cover [7].

Batik is one of the traditional fabrics and cultural heritage of Indonesia with various motifs according to their respective regions. The motif is the main element in batik, because the uniqueness of batik is from the motif itself. The motifs on batik cloth circulating in the market include geometric motifs, flora motifs, fauna motifs, figurative motifs, and there are also narrative motifs. These motifs lead to figurative, decorative, natural, geometric and stylized styles. Batik motifs on 
the market are generally dominated by flora and fauna motifs. On October 2, 2019, batik has been designated as an Intangible Cultural Heritage of Humanity by UNESCO. This is certainly good news not only for the government but also for Indonesian citizens, therefore, continuous research needs to be done to preserve it even though it has become a common research topic.

The development of modern batik has entered the realm of collaboration between batik science and technology, one of which is producing a new motif variant product. In the past, batik artists to create a batik motif required special skills and a long time. Recently computers were able to produce many ordinary images turned into works of art. This is done by a technology called artificial intelligence (AI). In recent months, someone named Christie presented AI artwork that was sold at one of their auctions for over $\$ 430,000$ [8]. When AI meets batik motifs, it can perform feature extraction on batik motifs. AI needs a model to extract features from batik motifs.

Reference [4] in 2015 Gatys made a model based on a neural network, namely the neural transfer style. Neural transfer style is a computer vision technique that takes two images, namely the so-called content image and the style reference image and then combines them all so that the resulting output image retains the core elements of the content image, but the output image looks like "brushed with paint" with the style of the image style reference. The neural transfer style is inspired by the power of convolutional neural networks (CNN). Neural network is tasked with extracting statistical features of the image related to content and style so that it can measure how well the resulting style transfers. Neural transfer style is a proposed model to create new batik motifs by extracting features from batik motifs.

The proposed contribution of this work is based on Gatys' neural style transfer algorithm with a few minor modifications. Generate new batik motif by utilizing asian games 2018 mascot as part of forming batik motif, not as batik users as in 1 . In this case, the content image is a silhouette of the 2018 Asian Games Mascot and style image is batik motif. This modifications for the proposed method is using a style image and then separating a style image for the silhouette pattern and a style image for the background image. This application uses a pre-trained network (VGGNET).

\section{METHOD}

Training the style transfer model to produce new image with other styles requires two steps:

A. The Pre-Trained Feature Extractor

Feature Extraction is used to find significant feature areas in an image depending on its intrinsic characteristics and application e.g. color, texture, etc. The Pre-Trained Feature Extractor based on CNN utilizes transfer learning using VGG Net to utilize a model that has been trained on a dataset to solve other similar problems by using it as a starting point, modifying and updating its parameters to suit the new dataset [9]Technically what cnn network did on can seen on fig 2.

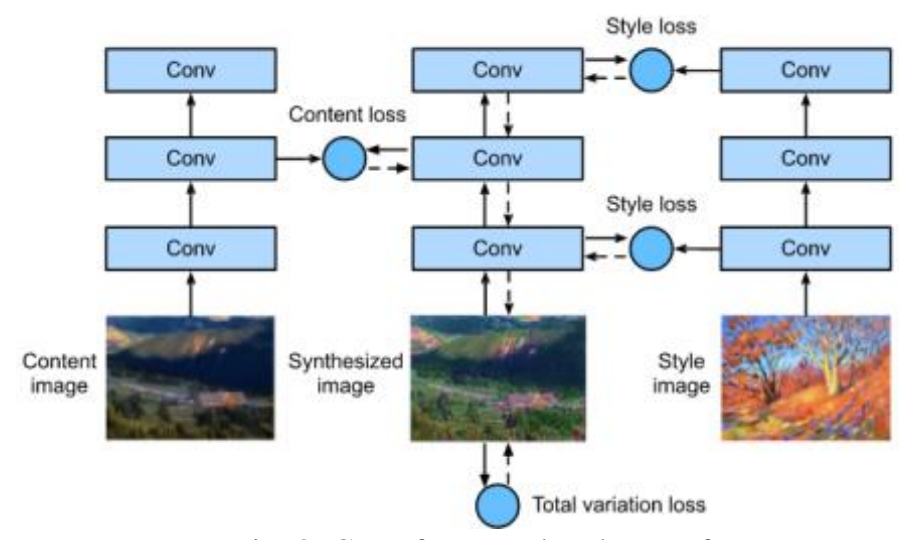

Fig. 2 CNN for neural style transfer

B. Style Transfer Network

Using a trained CNN, to extract image features and freeze its model parameters during training. This deep CNN uses multiple layers to extract hierarchical features for images. We can choose the output of some of these layers as content features or style features. The CNN contains three convolutional layers. The second layer displays the image features of the content, while the output of the first and third layers is used as a style feature. Next, it uses forward propagation (image showing the solid line direction) to calculate the style transfer loss function and backward propagation (image showing the dotted line direction) to update the model parameters, constantly updating the synthesis image. The synthesized image is the final image resulting from the neural transfer style. 


\author{
Impact Factor $7.39 \div$ Vol. 11, Issue 1, January 2022 \\ DOI: $10.17148 /$ IJARCCE.2022.11124
}

Stylish image quality is determined by the customizable loss functon. The loss function applies to both content images and images styles. Featured content extracted from styled images compared to original content images. The extracted style features are compared to those from the style image dataset. The VGG-16 model was pre-trained on the ImageNet dataset and used for the feature extractor. After going through each step the weights of the pre-trained feature extractor are correctedby weighing using a loss function, this can train the model to produce an output image with a lighter or heavier.Loss functions used in neural transfer styles generally have three parts:

- Loss content is used to make the synthetic image close to the content image with respect to the content features Information about any content image will be provided simply by activation of neurons at different layers. The deeper the layer, the more information it stores. It is calculated as the euclidean distance between the activation or representation of the content feature and the resulting image at different layers. Equation (1) for loss content.

$$
\text { L Content }^{(\vec{p}, \vec{x}, 1)}=\frac{1}{2} \sum_{i, j}^{n}\left(F_{i, j}^{l}-F_{i, j}^{l}\right) \ldots \ldots \ldots \ldots
$$

- Loss Style is used to make a synthetic image close to a stylized image in terms of stylistic features. Similar to loss content but the difference between the two is that the style of any image is not just represented as in the case of content, the image style is calculated using a gram matrix. Gram matrix serves to measure the layers of correlation between channels which will act as a measure of the style itself. Higher correlations give an idea of the style of an image. Gram Matrix formula in (2) and loss style formula in (3).

$$
\begin{aligned}
& G_{i, k}^{l}=\sum_{k}\left(F_{i, k}^{l}-F_{j, k}^{l}\right) \ldots \ldots \ldots \ldots \ldots \\
& \text { L Style }=\sum_{l} \sum_{i, j}\left(G_{i, j}^{s}-G_{i, j}^{p, l}\right)^{2} \ldots
\end{aligned}
$$

- Total variation loss helps reduce noise in the composition image. After noticing that loss style and loss content cause the output to have noise, then the total loss function (TV) is also included in the total loss in the NST. This total loss ensures continuity and spatial smoothness in the resulting image to avoid noise and over-pixel. Total loss is the sum of all losses. This weight determines how much impact the style or content has on the total loss. Simply put, if you want to have more stylistic influence than the content image on the synthesized image, give higher weight to the loss style which makes the total loss more dependent on the loss style and optimizing the total loss will focus more on optimizing the loss style.

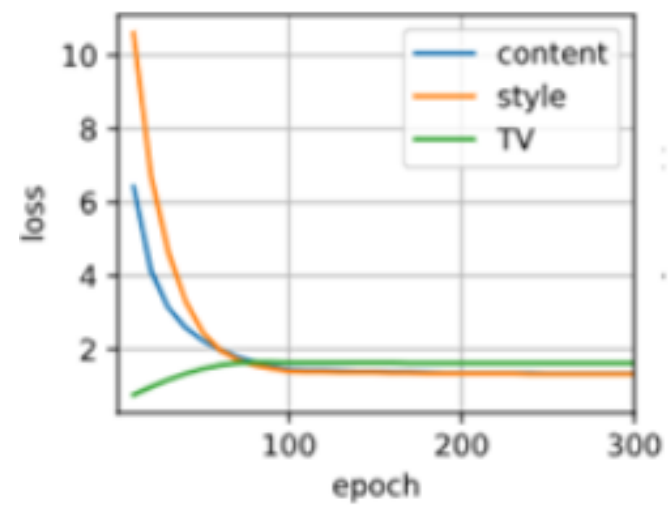

Fig. 3 Loss content, loss style and the total variation loss (TV)

If you want to make the resulting image more similar to the content image than the style image, then assign a weight to more influence of the content than the stylized image on the synthesis image, give higher weight to the loss content which makes the total loss more dependent on the loss content and optimizes the total loss will focus more on optimizing loss content.

The end of the model training is to display the style transfer model parameters to get a synthetic image. In Fig 3 it can be seen between the weight loss content, the weight loss style and the total weight variation loss (TV) on the epoch graph and loss function, good results on the transfer of style in the form of input image content combined with a style image produce a synthetic image that has a small density between loss content, loss style and total variation loss (TV). 


\section{III.RESULT AND DISCUSSION}

In our system, compute units consisted of a NVIDIA GT740M GPU and 16GB RAM. Experiments using 8 patterns of various batik motifs and 4 content images in silhouette images. In Fig 4 is an example of a style image and silhouette mascot image.
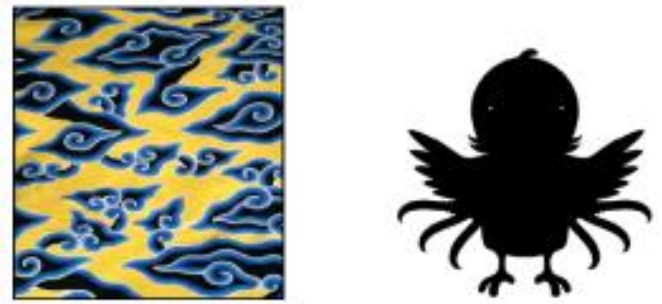

Fig. 4 A style image mega mendung motif and silhouette mascot image

In Fig 5, the algorithm process is tried on one of the mascot silhouette images and the Megamendung batik motif as a style image. The more iterations as carried out, the black silhouette as the content image will not be left and the image style giving a neat finishing. In this experiment, 10 iterations have been tried and the result are good enough for generating clear enough new batik motif. Silhouette color does not have to be black. Different colors can give different results. The black color of silhouette as the content image is neutral color of the silhouette color.

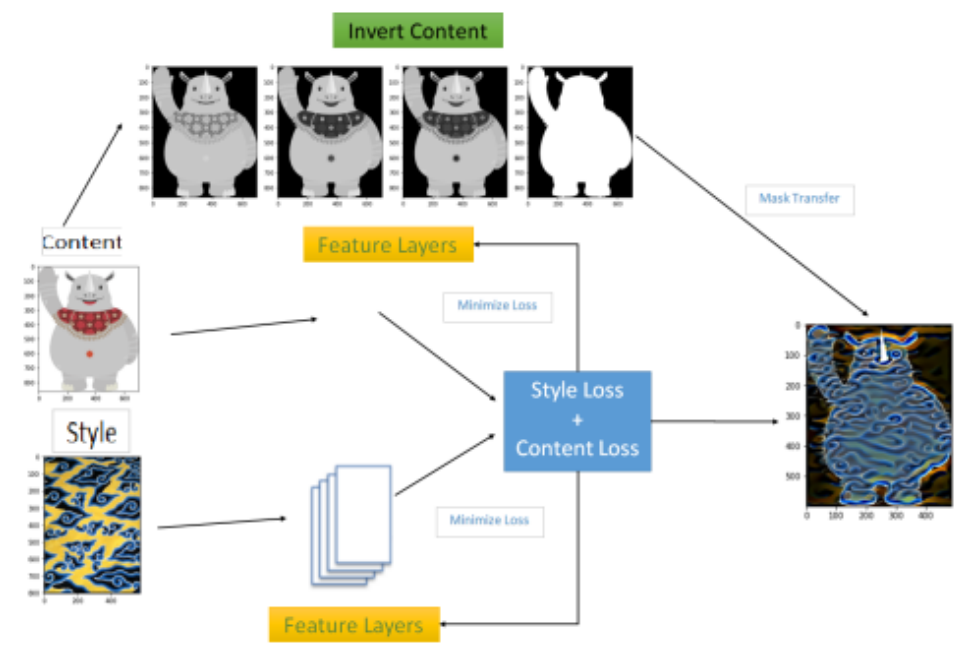

Fig. 5 Style transfer technique generate batik motif

The 2018 asian games mascot was changed to a silhouette image first. The reason for using silhouette images is to create easier to invert colors and apply image styles to invert content patterns and give more style effects to the content images. A new image are generated with a style that meets and completes the silhouette shape, the resulting pattern is parallel to the outline, then the generate image is given an additional background that can be adjusted through arguments.

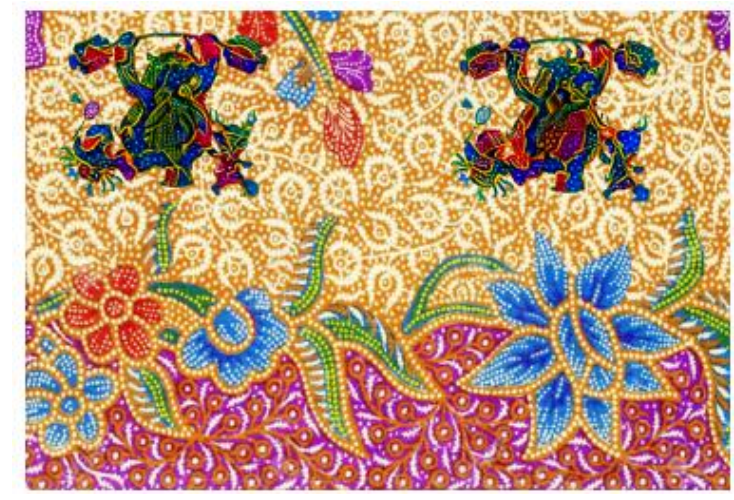

Fig. 6 A Asian Games 2018 mascot batik motif 
In Fig 6, is the result of the CNN algorithm applied to transfer style, generate asian games 2018 mascot for batik motif. Another result the Kawung Batik Motif from mascot called Bhinbhin in Fig 7 and Bhinbhin and flower motif in Fig 8.

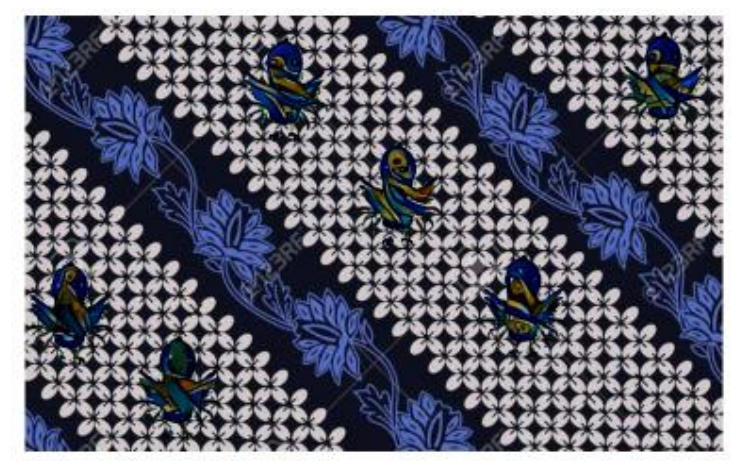

Fig.7 Kawung Batik Motif

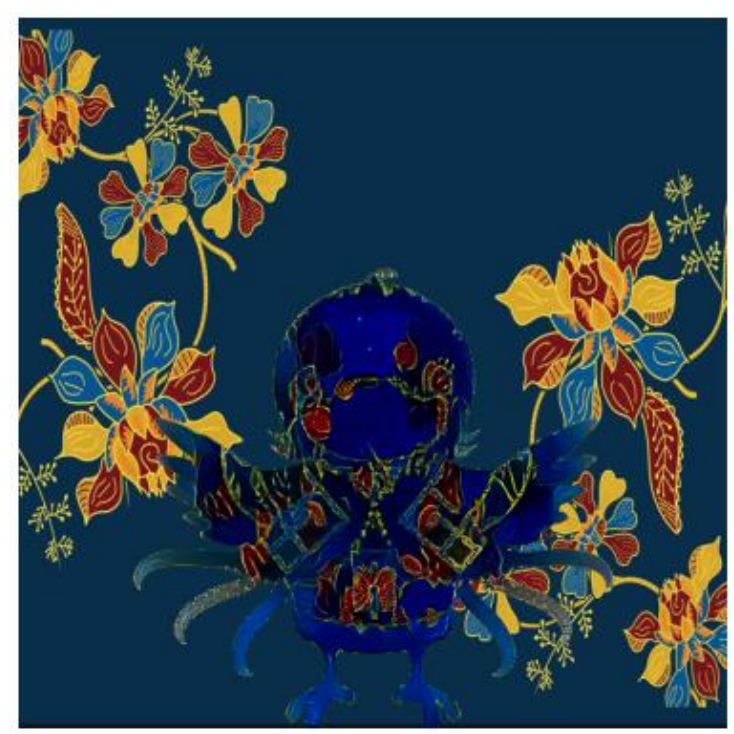

Fig. 8 Flower Batik Motif

\section{IV.CONCLUSION}

Neural style transfer is the process of creating art. The development of modern batik has entered the realm of collaboration between batik science and technology. Through this work Neural style transfer based on CNN architecture has perform in feature extraction on batik motifs even producing a new motif variant product. The paper have shown several results of variations of batik motifs from collaborations of existing batik motifs such as the kawung batik motif and Asian Games 2018 mascot image. The final evaluation of this work is subjective and very complex, involving individual tastes. Neural transfer style powers people around the world to experiment with their own creativity seeing the importance of style transfer in the commercial arts world and how art exists in the real world.

\section{ACKNOWLEDGMENT}

Thank to Indonesia Endowment Fund for Education (LPDP) Ministry of Finance Republic Indonesia have been made possible this research and presentation.

\section{REFERENCES}

[1] B. Gooch and A. Gooch, Non photorealistic rendering. 2001.

[2] T. Strothotte and S. Schlechtweg, Non-photorealistic computer graphics: modeling, rendering, and animation. 2002.

[3] C. P. Rosin and J, "Image and video-based artistic stylisation," Springer Science \& Business Media, vol. 42, 2012 . 
[4] C. V. S. L. A. Gatys, A. S. Ecker, M. Bethge, “A Neural Algorithm of Artistic Style,” 2015.

[5] T. Ariyani, "Inilah Wujud Nyata Hewan dan Batik yang Jadi Maskot Asian Games," 2018.

[6] W. Yudoseputro, Historiografi Seni Indonesia: Sebuah Pemikiran Terwujudnya Sejarah Seni Rupa Indonesia. 2010.

[7] Hidayaturrahman, "Style transfer: memindahkan style lukisan ke dalam gambar lain dengan menggunakan machine learning," Binus, 2020. https://socs.binus.ac.id/2020/11/22/style-transfer-memindahkan-stylelukisanke-dalam-gambar-lain-dengan-menggunakan-machine-learning/

[8] BBC Admin, "Portrait by AI program sells for \$432,000,” BBC News, 2018.

[9] K. Ramea, "Creating intricate art with neural style transfer," 2017. https://becominghuman.ai/creating-intricateart-with-neural-styletransfer-e5fee5f89481

\section{BIOGRAPHY}

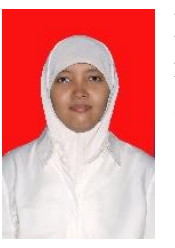

Rakhmi Khalida is a doctoral candidate in information technology at gunadarma university, her research interest are artificial inteligence, computer vision, and multimedia. Currently, she is teaching in informatic at Bhayangkara Jakarta Raya University Bekasi Indonesia. 